\section{EVALUATING IF COLONOSCOPIES ARE ESSENTIAL AFTER A DIVERTICULITIS DIAGNOSIS}

It was with great interest that we read the work of George $\mathrm{Ou}$ and colleagues ${ }^{1}$ regarding Colonoscopy after CT-diagnosed acute diverticulitis published June 1, 2015. While this paper explores a very relevant and topical subject there are a few issues we would like to highlight.

Although this paper was published in June 2015 the data were collected for this study from 2005 to 2010. This does make the data potentially outdated, and the authors provide no justification in the paper for what appear to be arbitrarily selected dates. There also appear to be some flaws in the methodology, which could produce some bias. The paper reports $79.6 \%$ of the patients received contrast with their CTs; this should have been standardized and the others excluded. There is also no mention of how many clinicians reported the CT scans or if it was a collaborative effort or the work of an individual radiologist, as we know this can differ from generalist to specialist radiologist. ${ }^{2}$

The paper does not have any followup data on $50.9 \%$ of the patients, which the authors have recognized as a limitation, but this could be a source of selection bias. The authors also state that $20.2 \%$ had premalignant polyps, which they give the endoscopic findings for but do not state if these were picked up on the CT scan. This could imply that not doing a colonoscopy after CT-diagnosed diverticulitis could potentially have led to 32 missed cancers. There is some evidence that CT scans miss around 1 in 29 colorectal cancers. ${ }^{3}$

The authors' state that among the patients found to have premalignant adenoma colonoscopy was of benefit, and they go on to suggest that it is conceivable this may have been missed without colonoscopy.

Overall, this study addresses a very relevant clinical question; however, given the points highlighted in this letter, there remains some doubt over the validity of the main conclusions. It would be worth repeating this study, rectifying these issues and those identified by the author. Until the question posed by this study is unequivocally answered, good practice would be to continue to follow-up patients with CT diagnosed diverticulitis with a colonoscopy.

Jonathan Herron, MSc; Sanjay Harrison, MBBS, MSc; Syed Casans, MBBS, PhD; Andrew Gilliam, MB; Luay Marrow, MB; Anna Mejsak, MD; Mia Charnley, MBBS; Adeel Aftab, MBBS

From the Darlington Memorial Hospital Surgical Journal Club, United Kingdom.

\section{DOI: $10.1503 /$ cjs.008915}

\section{References}

1. Ou G, Rosenfeld G, Brown J, et al. Colonoscopy after CT-diagnosed acute diverticulitis: Is it really necessary? Can $7 \mathrm{Surg}$ 2015;58:226-31.

2. Sickles EA, Wolverton DE, Dee KE. Performance parameters for screening and diagnostic mammography: specialist and general radiologists. Radiology 2002;224:861-9.

3. Atkin $W$, et al. Computed tomographic colonography versus colonoscopy for investigation of patients with symptoms suggestive of colorectal cancer (SIGGAR): a multicentre randomised trial. Lancet 2013;381:1194-202.

\section{AUthOR RESPONSE}

We appreciate the insightful comments from Herron and colleagues and the opportunity to expand on a few points.

Our retrospective study ${ }^{1}$ demonstrates that the prevalence of malignancy among patients with diverticu- litis diagnosed on high-resolution (64-slice) CT scan was $1.4 \%$, similar to that of average-risk population. ${ }^{2}$

The data period was chosen based on the time when high-resolution CT came into widespread use. Since then, 64-slice CT has remained the practice standard at our centre as well as at many others, thus maintaining the applicability of our results.

Ideally, contrast-enhanced CT should be used when acute diverticulitis is suspected, but this is often limited by contrast allergies and impaired renal function in practice. Although the quality of the studies are affected by lack of contrast, radiologists can still make the appropriate interpretations based on the clinical context provided by requesting clinician. We fear that excluding patients who were unable to receive contrast would have introduced substantial selection bias. To ensure that the included patients had findings consistent with acute diverticulitis, all of the CT scans in this study were retrospectively reviewed by a single radiologist specialized in abdominal imaging.

The premalignant findings in 23 patients were not specifically compared with the CT scans in this study. Unlike CT colonography, which invovles bowel preparation to rid of fecal matter and enteral contrast to distend the colon as well as to enhance the appearance of polyps, plain CT scan is not designed to assess intraluminal pathology.

None of the 23 patients with premalignant findings had undergone colorectal cancer (CRC) screening in the form of endoscopy (it was unknown if they had previous screening in the form of annual fecal occult blood tests) despite a mean age of 61.5 years owing to opportunistic screening being the primary strategy 
at that time. This finding, together with the fact that all 4 patients with malignancy also did not have previous CRC screening, underscores the importance of age-appropriate screening. We therefore recommend endoscopy-naive patients undergo follow-up endoscopic evaluation. On the other hand, if a patient already had high-quality colonoscopy with no evidence of polyp within a reasonable time before diverticulitis was diagnosed, it is conceivable that a repeat colonoscopy would be redundant.

One of the strengths of this study is the use of a provincial cancer registry to capture any CRC that may have risen since the diagnosis of acute diverticulitis. Absence of additional cases of CRC in the registry among those who did not have follow-up endoscopy lends support to the idea that not all patients with acute diverticulitis require follow-up endoscopy to rule out underlying malignancy.

Based on the results of our study, we recommend selective endoscopic evaluation in the following patient populations after a diagnosis of acute diverticulitis on high-resolution CT scan: patients $\geq 50$ years of age who are due for CRC screening/polyp surveillance in the form of colonoscopy based on recommended intervals, ${ }^{3}$ and those with suspicious CT findings, such as a mass lesion with obstruction.

\section{George Ou, MD; Greg Rosenfeld, MD; Brian Bressler, MSc, MD}

From the Department of Medicine, Division of Gastroenterology, St. Paul's Hospital, University of British Columbia, Vancouver, BC

DOI: $10.1503 /$ cjs.016515

\section{References}

1. Ou G, Rosenfeld G, Brown J, et al. Colonoscopy after CT-diagnosed acute diverticulitis: Is it really necessary? Can 7 Surg 2015;58:226-31.

2. Niv Y, Hazazi R, Levi Z, et al. Screening colonoscopy for colorectal cancer in asymptomatic people: a meta-analysis. $\mathrm{Dig}$ Dis Sci 2008;53:3049-54.

3. Lieberman DA, Rex DK, Winawer SJ, et al. Guidelines for colonoscopy surveillance after screening and polypectomy: a consensus update by the US Multi-Society Task Force on Colorectal Cancer. Gastroenterology 2012;143:844-57.

\section{OPERATIVE OR NONOPERATIVE MANAGEMENT OF HINCHEY III PURULENT ACUTE DIVERTICULITIS?}

We read with interest the article by Dr. Vennix and colleagues ${ }^{1}$ published in Lancet that rekindles the debate on management of severe acute diverticulitis (Hinchey III); the lower early morbidity of surgical resection reported by the authors definitely challenges the recent trend toward mini-invasive management by laparoscopic lavage (LL).,3

Interestingly, in the authors' whole series, major morbidity and 12-month mortality reached $30 \%$ and $11 \%$ (14\% in the resection group [RG]), respectively. Overall, patients underwent 157 operations (88 primary surgeries, 40 reoperations and 29 stoma reversals), accounting for a ratio of 1.8 operations per patient (1.9 in the RG). Moreover, $52 \%$ of patients underwent ileo-/colostomy (68\% in the RG), which was never reversed in $27 \%$ of cases. Finally, $15 \%$ of patients had fascial dehiscence within 1 year.

Also considering that patients with stercoral peritonitis (Hinchey IV) were excluded, results of surgery in the studied population seem poor and possibly caused by the emergency setting, rather than the purpose of surgery (resection s. nonresection). Admitting that LL is not superior because no difference is recorded between the 2 groups does not mean that performing an emergency sigmoidectomy is the best option in a septic patient with an ongoing acute peritonitis. The real, upcoming question seems to be whether Hinchey III patients (whose results are poor regardless of the performed procedure) really need emergency surgery. Since the study does not include a conservative management group, efficacy of antibiotics alone is not assessed.

We recently reported a $92 \%$ successful conservative management of hemodynamically stable patients with diverticulitis-associated pneumoperitoneum and no diffuse colonic perforation at CT $(82 \%$ and $72 \%$ presenting free intraperitoneal fluid and clinical signs of diffuse acute peritonitis, respectively). ${ }^{4}$ None died, 3 were reoperated and 7 required percutaneous drainage, which was considered a successful, nonoperative management, and we concluded that most non-Hinchey IV patients may be managed conservatively. Moreover, only 19 patients underwent delayed elective sigmoidectomy (with 2 reoperations), whereas 17 patients completely avoided surgery, with an overall ratio of 0.6 operations per patient.

In accordance with Vennix and coleagues, we believe that an accurate preoperative diagnosis should improve Hinchey III patient selection, not to undergo laparoscopic drainage, but rather to avoid an unnecessary surgery.

Randomized multicentre trials, comparing a surgical and a conservative approach to patients affected by non-Hinchey IV acute diverticulitis are needed to assess if, in this class of patients, less is more.

\section{Renato Costi, MD, PhD; Alban Zarzavadjian le Bian, $\mathrm{MD}$, PhD; Claude Smadja, MD, PhD; Vincenzo Violi, MD, PhD}

From the Dipartimento di Scienze Chirurgiche, Università degli Studi di Parma, Parma, Italia (Costi); the Service de Chirurgie Digestive et Carcinologique, Hôpital Lariboisière, Paris, Assistance Publique Hôpitaux de Paris, Université Paris VII "Diderot", France (Costi); the Service de Chirurgie Digestive, Hôpital "Simone Veil", Eaubonne, France (Zarzavadjian le Bian); and the Service de Chirurgie Digestive, Hôpital "Antoine Béclère", Clamart, Assistance Publique - Hôpitaux de Paris, Université Paris XI, France (Smadja).

\section{DOI: $10.1503 /$ cjs.015315}

\section{References}

1. Vennix S, Musters GD, Mulder IM, et al. Laparoscopic peritoneal lavage or sigmoidectomy for perforated diverticulitis with purulent peritonitis: a multicentre, parallel-group, randomised, open-label trial. Lancet 2015;386:1269-77.

2. Bretagnol F, Pautrat K, Mor C, et al. Emergency laparoscopic management of perforated sigmoid diverticulitis: a promising alternative to more radical procedures. 7 Am Coll Surg 2008;206:654-7.

3. Rossi GL, Mentz R, Bertone S, et al. Laparoscopic peritoneal lavage for Hinchey III diverticulitis: is it as effective as it is applicable. Dis Colon Rectum 2014;57:1384-90. 
4. Costi R, Cauchy F, Le Bian A, et al. Challenging a classic myth: pneumoperitoneum associated with acute diverticulitis is not an indication for open or laparoscopic emergency surgery in hemodynamically stable patients. A 10-year experience with a nonoperative treatment. Surg Endosc 2012; 26:2061-71.

\section{Re: RecurRence of INGUINAL HERNIAS REPAIRED IN A LARGE SURGICAL SPECIALTY HOSPITAL AND GENERAL HOSPITALS IN ONTARIO, Canada}

It is extremely rare, for an article dealing with the lowly hernia to provoke a tremor in the world of surgery. Malik and colleagues ${ }^{1}$ have just managed that seismic quality and they are to be congratulated and celebrated.

For 20 years, synthetic meshes have become the mainstay of hernia surgery, thanks to an industry that fostered conferences, societies and free courses, but also flagrant and undaunted marketing. The drawbacks of meshes were always vague and nebulous. ${ }^{2,3}$ The current generation of surgeons can truly be said to have been formed by the industry!

Mesh-based repairs are touted as the ideal in the guidelines of the European, Danish and Swedish Hernia Societies. ${ }^{4}$ The American Hernia Society has found no reason to disapprove.

The Shouldice repair, a pure tissue repair, rated a mention only when infected mesh was removed! Sadly, no one performs or knows how to perform a Shouldice repair outside Thornhill. A repair which, barely 20 years ago, was considered the "gold standard."

The world literature is now replete with publications on chronic postherniorrhaphy inguinodynia, a condition unknown before the introduction of mesh. In 1964, in Nyhus' classic hernia, postherniorrhaphy pain did not rate a mention in its index. ${ }^{5}$ In Ponka's equally excellent book, pain is mentioned in half a column as "uncommon" and due to "scar tissue" (1980). ${ }^{6}$

Copious publications are now firming up the statistics on postoperative complications of mesh: $11 \%$ of patients will have a history of severe chronic postoperative inguinodynia severe enough to be detrimental to their quality of life. Another group of patients (3\%-4\%) will suffer irreversible dysejaculation, which only 20 years ago, without mesh, had an incidence of 1 in 2500 cases and was reversible! A hundred-fold (or $10000 \%$ ) increase. Another 10\% will manifest severe testicular pain secondary to mesh erosion of the vas, which in some cases will require an orchidectomy. The specter of infertility has not been an issue, unless there is contact between mesh and vas (as in a Lichtenstein or laparoscopic approach) in a young adult with bilateral repairs and who may consider a family 10 years down the road. The delay in reaching the vas lumen is 7-10 years (unpublished data). Transmigration into adjacent organs are commonly reported but not systematically quantified with any accuracy through industrial surveillance. With such evidence, would a "duly informed" patient consent to mesh-based surgery?

To answer our respected authors, mesh is used in $3 \%$ of the cases at the Shouldice Hospital. Around the world, mesh is used in $90 \%-97 \% .7$

The better results of the Shouldice repair are not due to legerdemain. Their surgeons truly know anatomy. More so than surgeons who do an average of 50 cases a year. In the Swedish registry, $50 \%$ of the surgeons did fewer than 7 cases a year. The hackneyed aphorism with vendors that... " with mesh, you do not need to know anatomy" is simply untrue.

Professor Volcker Schumpelick, Editor in Chief of the Hernia, in his address to the American Hernia Society (2005) stated that "despite the introduction of mesh and laparoscopy, there has been no reduction in the incidence of hernia recurrences in the last 30 years. That incidence worldwide is $14 \% . "$ Why are the European guidelines rushing to be launched as World guidelines?

This thorough, objective, generously followed, massive population- based analysis by our Toronto colleagues has already reached Hernia and the European Hernia Society. The "tailored approach" concept is rather new. This paper will help nail that merciful concept. The figure estimated at $10 \%-20 \%$ could be brought to a mere $5 \%$ with simple emphasis of anatomy. The Shouldice Hospital already demonstrated, 20 years ago, that $1 \%-5 \%$ is the magic number depending on the type of hernia. ${ }^{9}$

This paper will become a classic, cited beyond the wildest dreams of its authors. It will awaken many residents to ask why it takes 4-6 years of surgical training if the industry can do it, through vendors, in a matter of minutes.

I would like to think that my learned and respected colleagues of the University of Toronto have revealed a good omen for a return to a saner algorithm and a harbinger of what I like to call a timely revival of a "greener operation."

\section{Robert Bendavid, MD}

From the Department of Surgery, Shouldice Hospital \& University of Toronto, Thornhill, Ontario.

DOI: $10.1503 /$ cjs.017715

\section{References}

1. Malik A, Bell CM, Stukel TA, et al. Recurrence of inguinal hernias repaired in a large surgical specialty hospital and general hospitals in Ontario, Canada. Can 7 Surg 2016;59:19-25.

2. Bendavid R, Lou W, Grischkan D, et al. A mechanism of mesh-related postherniorrhaphy neuralgia. Hernia 2015.

3. Iakovlev V, Guelcher SG, Bendavid R. Degradation of polypropylene in vivo: a microscopic analysis of meshes explanted from patients. 7 Biomed Mater Res B Appl Biomater 2015; doi:10.1002

4. Simons MP, Aufenacker T, Bay-Nielsen et al. European Hernia Society guidelines on the treatment of inguinal hernias in adult patients. Hernia 2009;13:343-403.

5. Nyhus LM. Hernia JB Lippincott; 1964.

6. Ponka J. Hernias of the abdominal wall. W B Saunders; 1980:601-602.

7. Ferrante F, Rusconi A, Galimberti A. Hernia repair in the Lombardy region in 2000. Preliminary results. Hernia 2004;8:247-251.

8. Schumpelick V. Hernia repair. AHS Meeting San Diego Meeting, February 9-12, 2005 [Abstract 46-I].

9. Bendavid R. Prosthetics in hernia surgery: a confirmation. Postgraduate General Surgery. 1992;4:166-7. 